\title{
High Order Local Approximations to Derivatives in the Finite Element Method
}

\author{
By Vidar Thomée
}

\begin{abstract}
Consider the approximation of the solution $u$ of an elliptic boundary value problem by means of a finite element Galerkin method of order $r$, so that the approximate solution $u_{h}$ satisfies $u_{h}-u=O\left(h^{r}\right)$. Bramble and Schatz (Math. Comp., v. 31, 1977, pp. 94-111) have constructed, for elements satisfying certain uniformity conditions, a simple function $K_{h}$ such that $K_{h} * u_{h}-u=O\left(h^{2 r-2}\right)$ in the interior. Their result is generalized here to obtain similar superconvergent order interior approximations also for derivatives of $u$.
\end{abstract}

1. Introduction. Assume that $u$ is a smooth function defined in $\Omega \subset R^{N}$. Assume also that one is given an approximation $u_{n}$ which belongs to a subspace $S_{n}$ (depending on a small parameter $h$ ) of $L_{2}(\Omega)$ such that (for notation, see Section 2)

$$
\left\|u-u_{h}\right\|_{\Omega} \leqslant C(u) h^{r}
$$

Such a situation arises when one solves a $2 m$ th order elliptic problem in a finite element space of order $r$ by Galerkin's method. In certain cases it is possible to derive estimates for $u-u_{h}$ in negative norms with a higher power of $h$, e.g.

$$
\left\|u-u_{h}\right\|_{-l, \Omega} \leqslant C(u) h^{r+l} .
$$

In the elliptic case referred to, this can sometimes be done by a duality argument, with $l=r-2 m$ if $r>2 m$.

In [2] Bramble and Schatz were able to show (this was carried out for second order equations, i.e. with $l=r-2$ ) that under certain uniformity assumptions on $\left\{S_{h}\right\}$ in the interior, it is possible to find an approximation of $u$ based on $u_{h}$ which is of this higher order also in $L_{2}\left(\Omega_{0}\right)$ where $\Omega_{0} \subset \subset \Omega$. Their approximation is a convolution $K_{n} * u_{n}$ where $K_{h}$ is a linear combination of translates of $B$-splines, and their result thus takes the form

$$
\left\|u-K_{h} * u_{n}\right\|_{\Omega_{0}} \leqslant C(u) h^{r+l} \text {. }
$$

The interior uniformity of $\left\{S_{h}\right\}$ is reflected in the fact that for any $\alpha$,

$$
\left\|\partial_{h}^{\alpha}\left(u-u_{h}\right)\right\|_{-l, \Omega_{0}} \leqslant C(u) h^{r+l},
$$

where $\partial_{h}^{\alpha}$ denote difference quotients. Under the assumption that in addition to (1), maximum norm estimates of the form

Received July 22, 1976.

AMS (MOS) subject classifications (1970). Primary 65N15, 65N30.

Copyright 0 1977, American Mathematical Society 


$$
\left|\partial_{h}^{\alpha}\left(u-u_{h}\right)\right|_{\Omega_{0}} \leqslant C(u) h^{r}
$$

are valid (such estimates hold in the elliptic case, cf. Bramble, Nitsche and Schatz [1]), Bramble and Schatz also showed that

$$
\left|u-K_{h} * u_{n}\right|_{\Omega_{0}} \leqslant C(u) h^{r+l}
$$

The purpose of this note is to derive similar approximations also for arbitrary derivatives of $u$, and in fact for any linear functional of $u$ which is continuous on some Sobolev space $W_{2}^{n}\left(\Omega_{0}\right)$. In deriving these results we also give alternative proofs of the results by Bramble and Schatz.

Our first main result, Theorem 1 below, shows that an arbitrary derivative of the smooth function $u$ can be approximated in $L_{2}\left(\Omega_{0}\right)$ by a local average of the corresponding difference quotient of the approximating function $u_{n}$. Hereby the error can be bounded by $O\left(h^{2 p}\right)$ plus a finite sum of terms of the form of the lefthand side in (1), where $p$ and $l$ are arbitrary given positive integers. Using this result we show in Theorem 2 that the value at $u$ of any bounded linear functional $\Lambda$ on some $W_{2}^{n}\left(\Omega_{0}\right)$ can be approximated to the same degree as in Theorem 1 by a linear functional $\Lambda_{h}$ acting on $u_{n}$. Finally, in Theorem 3, we show the analogue of Theorem 1 for the maximum norm. Now interior maximum norms of certain difference quotients of the error have to be included in the error bound.

Our results are not restricted to the solution of elliptic problems by Galerkin's method but may be applied in any situation where negative norm (and for Theorem 3 , maximum norm) interior estimates are available for difference quotients of the error. Such estimates were obtained recently for second order parabolic equations in Bramble, Schatz, Thomée and Wahlbin [3]. The conclusion is therefore that in the context of that paper, $O\left(h^{2 r-2}\right)$ approximations can be obtained in the interior by local averaging, not only for the solution itself, which was shown in [3], but also for any derivative of the solution.

2. Notation. For $\Omega$ a domain in $R^{N}$ and $s$ a nonnegative integer we set for $v \in W_{2}^{s}(\Omega)$, with $\|\cdot\|_{\Omega}$ the norm in $L_{2}(\Omega)$,

$$
\|v\|_{s, \Omega}=\left(\sum_{|\alpha| \leqslant s}\left\|D^{\alpha} v\right\|_{\Omega}^{2}\right)^{1 / 2},
$$

and for $v \in L_{2}(\Omega)$,

$$
\|v\|_{-s, \Omega}=\sup _{w \in C_{0}^{\infty}(\Omega)} \frac{(v, w)}{\|w\|_{s, \Omega}} .
$$

Note that for any integer $s,\|\cdot\|_{s, \Omega}$ increases with $\Omega$ and that $\|\cdot\|_{s, R^{N}}$ is equivalent to

$$
\|v\|_{s}=\left(\int_{R}\left(1+|\xi|^{2}\right)^{s}|\hat{v}|^{2} d \xi\right)^{1 / 2} .
$$

Notice also that for supp $v \subset \Omega_{0} \subset \subset \Omega_{1} \subset \subset \Omega_{2}$ we have for positive $s,\|v\|_{-s, \Omega_{2}} \leqslant$ $C\|v\|_{-s, \Omega_{1}}$, so that the two norms are then equivalent. For if $\varphi \in C_{0}^{\infty}\left(\Omega_{1}\right)$ and $\varphi \equiv 1$ on $\Omega_{0}$ we have 


$$
\|v\|_{-s, \Omega_{2}}=\sup _{w \in C_{0}^{\infty}\left(\Omega_{2}\right)} \frac{(\varphi v, w)}{\|w\|_{s, \Omega_{2}}} \leqslant C \sup _{w \in C_{0}^{\infty}\left(\Omega_{2}\right)} \frac{(v, \varphi w)}{\|\varphi w\|_{s, \Omega_{2}}} \leqslant C\|v\|_{-s, \Omega_{1}}
$$

In addition to these $L_{2}$ based norms we shall use the maximum norm

$$
|v|_{\Omega}=\sup _{x \in \Omega}|v(x)|
$$

and for $v \in C^{s}(\bar{\Omega})$,

$$
|v|_{s, \Omega}=\max _{|\alpha| \leqslant s}\left|D^{\alpha} v\right|_{\Omega}
$$

Let $\psi_{(l)}$ denote the $B$-spline of order $l$, that is the convolution $\chi * \cdots * \chi$ with $l$ factors, where $\chi$ is the characteristic function of $[-1 / 2,1 / 2]$. For $\alpha=$ $\left(\alpha_{1}, \ldots, \alpha_{N}\right)$ let

$$
\psi_{(\alpha)}(x)=\prod_{j=1}^{N} \psi_{\left(\alpha_{j}\right)}\left(x_{j}\right)
$$

denote the $B$-spline of order $\alpha$ in $R^{N}$. In particular, with $\epsilon=(1, \ldots, 1), \psi_{(l \epsilon)}$ denotes the $B$-spline of the same order $l$ in each variable. We set $\psi_{(\alpha), h}(x)=$ $h^{-N} \psi_{(\alpha)}\left(h^{-1} x\right)$.

We define the difference quotient operator $\partial_{h}^{\alpha}$ by

$$
\partial_{h}^{\alpha}=\partial_{h, 1}^{\alpha} \cdots \partial_{h, N}^{\alpha}, \quad \text { with } \partial_{h, j} v(x)=h^{-1}\left(v\left(x+1 / 2 h e_{j}\right)-v\left(x-1 / 2 h e_{j}\right)\right) .
$$

Notice that for the Fourier transforms of $\psi_{(\alpha), h}$ and $\partial_{h}^{\alpha} v$ we have

$$
\hat{\psi}_{(\alpha), h}(\xi)=\hat{\psi}_{(\alpha)}(h \xi)=\prod_{j=1}^{N}\left(\frac{\sin \left(1 / 2 h \xi_{j}\right)}{1 / 2 h \xi_{j}}\right)^{\alpha}
$$

and

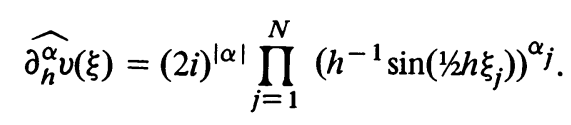

Let $a \in C^{\infty}\left(R^{N}\right)$. We recall that $a$ is said to be a Fourier multiplier on $L_{\infty}$ or $a \in M=M_{\infty}$ if

$$
M(a)=\sup \left\{\left|F^{-1}(a \hat{v})\right|_{R^{N}} ; v \in C_{0}^{\infty}\left(R^{N}\right),|v|_{R^{N}} \leqslant 1\right\}<\infty,
$$

where $F^{-1}$ denotes the inverse Fourier transform (for related material, cf.,e.g. [4]). We shall use below that $a(h \xi) \in M$ if $a \in M$, with $M(a(h \cdot))$ independent of $h$ so that

$$
\left|F^{-1}(a(h \xi) \hat{v})\right|_{R} N \leqslant M(a)|v|_{R^{N}} .
$$

We shall also use the facts that $M$ is closed under multiplication, that any trigonometric polynomial and $\psi_{(\alpha)}$ belong to $M$ (in fact, $M\left(\psi_{(\alpha)}\right)=1$ ), that $C_{0}^{\infty} \subset M$ and further that if $\varphi$ is a $C^{\infty}$ function on the real line with $\varphi^{(l)}\left(\xi_{j}\right)=O\left(\left|\xi_{j}\right|^{-b-l}\right)(b>0, l=0,1)$, for large $\left|\xi_{j}\right|$, then $\varphi$ belongs to $M$ in one and, as a consequence, also in $N$ dimensions. 
3. The Local Averaging Operators. We shall now turn to the construction of the kernels of the averaging operators used in our main results. The first step is the definition of certain one-dimensional trigonometric polynomials.

Lemma 1. Let $q \geqslant 1, p \geqslant 1$. Then there exists a unique trigonometric polynomial $k_{q, p}$ of order $p-1$ such that

$$
k_{q, p}(\sigma) \hat{\chi}(\sigma)^{q}=1+O\left(\sigma^{2 p}\right) \text { as } \sigma \rightarrow 0 .
$$

Proof. Setting $\tau=\sin 1 / 2 \sigma$ we obtain for small $\tau$ or $\sigma$,

$$
\hat{\chi}(\sigma)^{-q}=\left(\frac{\arcsin \tau}{\tau}\right)^{q}=\sum_{j=0}^{\infty} \gamma_{q, j} \tau^{2 j} .
$$

We may therefore choose

$$
k_{q, p}(\sigma)=\sum_{j=0}^{p-1} \gamma_{q, j}\left(\sin \frac{1}{2} \sigma\right)^{2 j}=\sum_{j=0}^{p-1} \gamma_{q, j} 2^{-j}(1-\cos \sigma)^{j}
$$

The uniqueness follows at once since if $k_{1}$ and $k_{2}$ both satisfy (3) then $k_{1}-k_{2}$ is a trigonometric polynomial of order $p-1$ which vanishes of order $2 p$ at the origin and hence has to vanish identically.

For $\alpha=\left(\alpha_{1}, \ldots, \alpha_{N}\right)$ with $\alpha_{j}>0$ and $p \geqslant 1$ we now define the $N$-dimensional trigonometric polynomial

$$
k^{(\alpha, p)}(\xi)=\prod_{j=1}^{N} k_{\alpha_{j}, p}\left(\xi_{j}\right)=\sum_{\gamma} k_{\gamma}^{(\alpha, p)} e^{-i\langle\xi, \gamma\rangle}, \quad \xi, \gamma \in R^{N}
$$

Using the coefficients of this polynomial and the $B$-splines of order $\alpha$ we set

Notice that by (3),

$$
K^{(\alpha, p)}(x)=\sum_{\gamma} k_{\gamma}^{(\alpha, p)} \psi_{(\alpha)}(x-\gamma)
$$

$$
\begin{aligned}
\hat{K}^{(\alpha, p)}(\xi) & =k^{(\alpha, p)}(\xi) \hat{\psi}_{(\alpha)}(\xi)=\prod_{1}^{N}\left(k_{\alpha_{j}, p}\left(\xi_{j}\right) \hat{\chi}\left(\xi_{j}\right)^{\alpha}\right) \\
& =1+O\left(|\xi|^{2 p}\right) \quad \text { as } \xi \longrightarrow 0
\end{aligned}
$$

We also set

$$
K_{h}^{(\alpha, p)}(x)=h^{-N} K^{(\alpha, p)}\left(h^{-1} x\right)
$$

The order relation near the origin in (4) is the basis for proving the following approximation estimate for convolution by $K_{h}^{(\alpha, p)}$.

LEMmA 2. Let $\alpha=\left(\alpha_{1}, \ldots, \alpha_{N}\right)$ with $\alpha_{j}>0$ and $p \geqslant 1$ be given. Then for $\Omega_{1} \subset \subset \Omega_{2}$ there is a constant $C$ such that for small $h$,

$$
\begin{gathered}
\left\|v-K_{h}^{(\alpha, p)} * v\right\|_{\Omega_{1}} \leqslant C h^{2 p} \mid\|v\|_{2 p, \Omega_{2}}, \\
\left|v-K_{h}^{(\alpha, p)} * v\right|_{\Omega_{1}} \leqslant C h^{2 p}|v|_{2 p, \Omega_{2}} .
\end{gathered}
$$


Proof. Since by (4),

$$
\left|1-\hat{K}^{(\alpha, p)}(h \xi)\right| \leqslant C h^{2 p}|\xi|^{2 p} \text { for } \xi \in R^{N},
$$

we obtain at once by Parseval's relation, for $v \in C_{0}^{\infty}\left(R^{N}\right)$,

$$
\left\|v-K_{h}^{(\alpha, p)} * v\right\|_{0} \leqslant C h^{2 p}\|v\|_{2 p} .
$$

The estimate (5) now follows by applying this inequality to $\omega v$ where $\omega \in C_{0}^{\infty}\left(\Omega_{2}\right)$ and $\omega \equiv 1$ in a neighborhood of $\bar{\Omega}_{1}$.

Setting $\widetilde{k}_{j}(\sigma)=k_{\alpha_{j}, p}(\sigma) \hat{\chi}(\sigma)^{\alpha}$, we find since $\left(1-\widetilde{k}_{j}\left(\xi_{j}\right)\right) \xi_{j}^{-2 p} \in M$ that (with $\left.D_{j}=\partial / \partial x_{j}\right)$

$$
\begin{aligned}
\left|F^{-1}\left(\left(1-\tilde{k}_{j}\left(h \xi_{j}\right)\right) \hat{v}\right)\right|_{R^{N}} & =h^{2 p} \mid F^{-1}\left(\left(1-\widetilde{k}_{j}\left(h \xi_{j}\right)\right)\left(h \xi_{j}\right)^{-2 p} \widehat{\left.D_{j}^{2 p} v\right)\left.\right|_{R^{N}}}\right. \\
& \leqslant C h^{2 p}\left|D_{j}^{2 p} v\right|_{R^{N}} .
\end{aligned}
$$

Writing

$$
1-\hat{K}^{(\alpha, p)}(h \xi)=\sum_{j=1}^{N} \prod_{l<j} \widetilde{k}_{l}\left(h \xi_{l}\right)\left(1-\widetilde{k}_{j}\left(h \xi_{j}\right)\right),
$$

and noticing that clearly also $\widetilde{k}_{j} \in M$, we conclude

$$
\left|v-K_{h}^{(\alpha, p)} * v\right|_{R^{N}}=\left|F^{-1}\left(\left(1-\hat{K}^{(\alpha, p)}(h \xi)\right) \hat{v}\right)\right|_{R^{N}} \leqslant C h^{2 p}|v|_{2 p, R^{N}}
$$

from which (6) follows as above by application to $\omega v$.

In addition to the function $K_{h}^{(\alpha, p)}$ we shall use the associated function defined for $\alpha \geqslant 0, l \geqslant 1, p \geqslant 1$ by

$$
\widetilde{K}_{h}^{(\alpha, l, p)}(x)=\sum_{\gamma} k_{\gamma}^{(\alpha+l \epsilon, p)} \psi_{(l \epsilon), h}(x-\gamma h) .
$$

Recall that derivatives of splines of higher order are difference quotients of splines of lower order. In particular, more precisely,

$$
D^{\alpha} \psi_{(\alpha+l \epsilon), h}=\partial_{h}^{\alpha} \psi_{(l \epsilon), h},
$$

so that with the above notation

$$
D^{\alpha} K_{h}^{(\alpha+l \epsilon, p)}=\partial_{h}^{\alpha} \widetilde{K}_{h}^{(\alpha, l, p)} .
$$

4. Main results. We can now prove the first of our main results.

THEOREM 1. Let $\alpha$ be a given multi-index, let $l \geqslant 1$ and $p \geqslant 1$. Then, for $\Omega_{0} \subset \subset \Omega_{1} \subset \subset \Omega_{2}$ there is a constant $C$ such that for $h$ small, $u \in W_{2}^{2 p+|\alpha|}\left(\Omega_{1}\right)$ and $u_{h} \in L_{2}\left(\Omega_{2}\right)$,

$$
\begin{aligned}
& \left\|D^{\alpha} u-\widetilde{K}_{h}^{(\alpha, l, p)} * \partial_{h}^{\alpha} u_{h}\right\|_{\Omega_{0}} \\
& \quad \leqslant C\left\{h^{2 p}\|u\|_{2 p+|\alpha|, \Omega_{1}}+\sum_{|\beta| \leqslant l}\left\|\partial_{h}^{\alpha+\beta}\left(u-u_{h}\right)\right\|_{-l, \Omega_{1}}\right\} .
\end{aligned}
$$


Proof. Notice that by (7),

$$
\tilde{K}_{h}^{(\alpha, l, p)} * \partial_{h}^{\alpha} v=\partial_{h}^{\alpha} \widetilde{K}_{h}^{(\alpha, l, p)} * v=D^{\alpha} K_{h}^{(\alpha+l \epsilon, p)} * v=K_{h}^{(\alpha+l \epsilon, p)} * D^{\alpha} v .
$$

We have by the triangle inequality

$$
\begin{aligned}
& \left\|D^{\alpha} u-\widetilde{K}_{h}^{(\alpha, l, p)} * \partial_{h}^{\alpha} u_{h}\right\|_{\Omega_{0}} \\
& \quad \leqslant\left\|D^{\alpha} u-K_{h}^{(\alpha+l \epsilon, p)} * D^{\alpha} u\right\|_{\Omega_{0}}+\left\|\tilde{K}_{h}^{(\alpha, l, p)} * \partial_{h}^{\alpha}\left(u-u_{h}\right)\right\|_{\Omega_{0}}=\mathrm{I}+\mathrm{II} .
\end{aligned}
$$

By Lemma 2,

$$
\mathrm{I} \leqslant C h^{2 p}\left\|D^{\alpha} u\right\|_{2 p, \Omega_{1}} \leqslant C h^{2 p}\|u\|_{2 p+|\alpha|, \Omega_{1}} .
$$

The proof is therefore completed by the following lemma.

Lemma 3. Let $l \geqslant 1$ and $\Omega_{0} \subset \subset \Omega_{1} \subset \subset \Omega_{2}$. Then there exists a constant $C$ such that for small $h$ and $v \in L_{2}\left(\Omega_{2}\right)$,

$$
\left\|\psi_{(l \epsilon), h} * v\right\|_{\Omega_{0}} \leqslant C \sum_{|\beta| \leqslant l}\left\|\partial_{h}^{\beta} v\right\|_{-l, \Omega_{1}} \text {. }
$$

Proof. We notice that for each $j$,

$$
\left(\frac{\sin \left(1 / 2 h \xi_{j}\right)}{1 / 2 h \xi_{j}}\right)^{2 l} \leqslant C \frac{1+\left(h^{-1} \sin \left(1 / 2 h \xi_{j}\right)\right)^{2 l}}{\left(1+\xi_{j}^{2}\right)^{l}}, \quad \xi_{j} \in R, h<1
$$

This is obvious for small $h \xi_{j}$ since then the left side is bounded above and the right side below. For large $h \xi_{j}$ and hence $\xi_{j}$ large the result is also immediate. Letting $\xi_{0}$ denote the component of $\xi$ with largest modulus, we conclude, since $|\hat{\chi}| \leqslant 1$,

$$
\hat{\psi}_{(l \epsilon)}(h \xi)^{2} \leqslant\left(\frac{\sin \left(1 / 2 h \xi_{0}\right)}{1 / 2 h \xi_{0}}\right)^{2 l} \leqslant C \frac{1+\left(h^{-1} \sin \left(1 / 2 h \xi_{0}\right)\right)^{2 l}}{\left(1+\xi_{0}^{2}\right)^{l}}
$$

$$
\leqslant C \frac{1+\sum_{j}\left(h^{-1} \sin \left(1 / 2 h \xi_{j}\right)\right)^{2 i}}{\left(1+|\xi|^{2}\right)^{l}}
$$

Parseval's relation therefore yields

$$
\begin{aligned}
\left\|\psi_{(l \epsilon), h} * v\right\|_{R^{N}} & \leqslant C\left\{\|v\|_{-l, R^{N}}+\sum_{j} \| \partial_{h}^{l e} j_{v \|_{-l, R} N}\right\} \\
& \leqslant C \sum_{|\beta| \leqslant l}\left\|\partial_{h}^{\beta} v\right\|_{-l, R^{N}} .
\end{aligned}
$$

An application of this inequality to $v \omega$, where $\omega \in C_{0}^{\infty}\left(\Omega_{1}\right)$ and $\omega \equiv 1$ in a neighborhood of $\Omega_{0}$ completes the proof.

Theorem 1 may now be used to produce approximations to $\Lambda u$ for any bounded linear functional on a Sobolev space on $\Omega_{0}$.

THEOREM 2. Let $l \geqslant 1, p \geqslant 1$ and $\Omega_{0} \subset \subset \Omega_{1} \subset \subset \Omega_{2}$. Let $\Lambda$ be a bounded linear functional on $W_{2}^{n}\left(\Omega_{0}\right)$ for some $n \geqslant 0$. Then there exists a constant $C$ and 
for sufficiently small $h$ a bounded linear functional $\Lambda_{h}$ on $L_{2}\left(\Omega_{1}\right)$ such that for $u \in$ $W_{2}^{2 p+n}\left(\Omega_{2}\right), u_{n} \in L_{2}\left(\Omega_{2}\right)$,

$$
\left|\Lambda u-\Lambda_{h} u_{n}\right| \leqslant C\left\{h^{2 p}\|u\|_{2 p, \Omega_{1}}+\sum_{|\alpha| \leqslant n ;|\beta| \leqslant l}\left\|\partial_{h}^{\alpha+\beta}\left(u-u_{h}\right)\right\|_{-l, \Omega}\right\} \text {. }
$$

Proof. By the Riesz representation theorem there is a $\varphi \in W_{2}^{n}\left(\Omega_{0}\right)$ such that with $(\cdot, \cdot)_{\Omega_{0}}$ the inner product in $L_{2}\left(\Omega_{0}\right)$,

$$
\Lambda u=(u, \varphi)_{w_{2}^{n}\left(\Omega_{0}\right)}=\sum_{|\alpha| \leqslant n}\left(D^{\alpha} u, D^{\alpha} \varphi\right)_{\Omega_{0}} .
$$

Setting

$$
\Lambda_{h} v=\sum_{|\alpha| \leqslant n}\left(\widetilde{K}_{h}^{(\alpha, l, p)} * \partial_{h}^{\alpha} v, D^{\alpha} \varphi\right)_{\Omega_{0}}
$$

we obtain

$$
\left|\Lambda u-\Lambda_{h} u_{h}\right| \leqslant\|\varphi\|_{n, \Omega_{0}} \sum_{|\alpha| \leqslant n}\left\|D^{\alpha} u-\widetilde{K}_{h}^{(\alpha, l, p)} * \partial_{h}^{\alpha} u_{h}\right\|_{\Omega_{0}},
$$

and the result follows by Theorem 1 .

In particular, in the case of the elliptic problem treated by Bramble and Schatz, we conclude by Theorem 2 and (1) that the approximating function $u_{h}$ contains enough information to produce a $O\left(h^{r+l}\right)$ approximation of $D^{\alpha} u(x)$ for any $\alpha$ and any $x \in \Omega$. The corresponding functional $\Lambda_{h}$ is of course not practical, and since also (2) holds, we therefore have use for the following direct interior maximum norm estimate.

THEOREM 3. Let $\alpha$ be a given multi-index, let $l \geqslant 1$ and $p \geqslant 1$. Then for $\Omega_{0}$ $\subset \subset \Omega_{1} \subset \subset \Omega_{2}$ there is a constant $C$ such that for $h$ small and $u \in C^{2 p+|\alpha|}\left(\Omega_{2}\right), u_{n}$ $\in C\left(\Omega_{2}\right)\left(\right.$ with $\left.N_{0}=[N / 2]+1\right)$,

$$
\begin{aligned}
& \left|D^{\alpha} u-\widetilde{K}^{(\alpha, l, p)} * \partial_{h}^{\alpha} u_{h}\right|_{\Omega_{0}} \\
& \qquad C\left\{h^{2 p}|u|_{2 p+|\alpha|, \Omega_{1}}+\sum_{|\beta| \leqslant l+N_{0}} \|\left.\partial_{h}^{\alpha+\beta}\left(u-u_{h}\right)\right|_{-l, \Omega_{1}}\right. \\
& \left.+h^{l} \sum_{|\beta| \leqslant l}\left|\partial_{h}^{\alpha+\beta}\left(u-u_{h}\right)\right|_{\Omega_{1}}\right\} .
\end{aligned}
$$

Proof. In the same way as in the proof of Theorem 1, using now the second part of Lemma 2, we obtain

$$
\left|D^{\alpha} u-\widetilde{K}_{h}^{(\alpha, l, p)} * \partial_{h}^{\alpha} u_{h}\right|_{\Omega_{0}} \leqslant C h^{2 p}|u|_{2 p+|\alpha|, \Omega_{1}}+\left|\widetilde{K}_{h}^{(\alpha, l, p)} * \partial_{h}^{\alpha}\left(u-u_{h}\right)\right|_{\Omega_{0}} .
$$

The result therefore follows as above by the following analogue of Lemma 3 .

LemmA 4. Let $l \geqslant 1$ and $\Omega_{0} \subset \subset \Omega_{1} \subset \subset \Omega_{2}$. Then there exists a constant $C$ such that for $h$ small and $v \in C\left(\Omega_{2}\right)$,

$$
\left|\psi_{(l \epsilon), h} * v\right|_{\Omega_{0}} \leqslant C\left\{\sum_{|\beta| \leqslant l+N_{0}}\left\|\partial_{h}^{\beta} v\right\|_{-l, \Omega_{1}}+h^{l} \sum_{|\beta| \leqslant l}\left|\partial_{h}^{\beta} v\right|_{\Omega_{1}}\right\} .
$$


Proof. As previously, it is sufficient to prove the result for $v \in C_{0}^{\infty}\left(\Omega_{1}\right)$. Let $\varphi \in C_{0}^{\infty}(-\pi, \pi)$ with $\varphi \equiv 1$ near the origin and set $\Phi(\xi)=\Pi_{1}^{N} \varphi\left(\xi_{j}\right)$. We may write, at $x \in \Omega_{0}$,

$$
\begin{aligned}
\psi_{(l \epsilon), h} * v & =F^{-1}\left(\hat{\psi}_{(l \epsilon)}(h \xi) \hat{v}\right) \\
& =F^{-1}\left(\hat{\psi}_{(l \epsilon)}(h \xi) \Phi(h \xi) \hat{v}\right)+F^{-1}\left(\hat{\psi}_{(l \epsilon)}(h \xi)(1-\Phi(h \xi)) \hat{v}\right)=\mathrm{I}+\mathrm{II} .
\end{aligned}
$$

Using Schwarz's inequality, we obtain

$$
\begin{aligned}
|\mathrm{I}| & =\left|\int\left(1+|\xi|^{2}\right)^{-N_{0} / 2} e^{-i\langle x, \xi\rangle}\left(1+|\xi|^{2}\right)^{N_{0} / 2} \hat{\psi}_{(l \epsilon)}(h \xi) \Phi(h \xi) \hat{v}(\xi) d \xi\right| \\
& \leqslant C\left\|\left(1+|\xi|^{2}\right)^{N_{0} / 2} \hat{\psi}_{(l \epsilon)}(h \xi) \Phi(h \xi) \hat{v}(\xi)\right\|_{R^{N}} .
\end{aligned}
$$

For $h \xi$ in the support of $\Phi(h \xi)$ we have

and hence by (8),

$$
1+|\xi|^{2} \leqslant C\left\{1+\sum_{j}\left(h^{-1} \sin \left(\frac{1}{2} h \xi_{j}\right)\right)^{2}\right\}
$$

$$
\begin{aligned}
& \left(1+|\xi|^{2}\right)^{N_{0} / 2} \hat{\psi}_{(l \epsilon)}(h \xi) \Phi(h \xi) \\
& \quad \leqslant C\left(1+|\xi|^{2}\right)^{-1 / 2}\left\{1+\sum_{j}\left(h^{-1} \sin \left(\frac{1}{2} h \xi_{j}\right)\right)^{2\left(l+N_{0}\right)}\right\}^{1 / 2} .
\end{aligned}
$$

We conclude

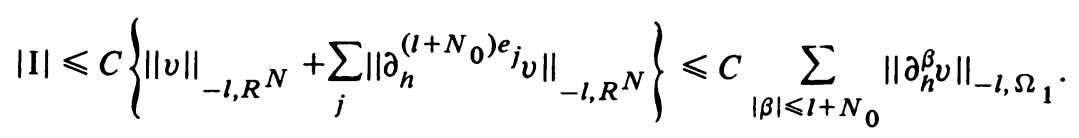

In order to estimate II we write

$$
\mathrm{II}=F^{-1}\left(\prod_{j} \hat{\chi}\left(h \xi_{j}\right)^{l} \sum_{j} \prod_{i<j} \varphi\left(h \xi_{i}\right)\left(1-\varphi\left(h \xi_{j}\right)\right) \hat{v}\right),
$$

and hence since $\hat{\chi}, \varphi$ and $\left(1-\varphi\left(\xi_{j}\right)\right) / \xi_{j}^{l}$ belong to $M$,

$$
\begin{aligned}
|\mathrm{II}| & \leqslant C \sum_{j}\left|F^{-1}\left(\left(1-\varphi\left(h \xi_{j}\right)\right) \hat{\chi}\left(h \xi_{j}\right) l \hat{v}\right)\right|_{R^{N}} \\
& =C^{l} \sum_{j} \mid F^{-1}\left(\left(1-\varphi\left(h \xi_{j}\right)\right)\left(h \xi_{j}\right)^{-l} \widehat{\left.\partial_{h}^{l j_{j}} v\right)\left.\right|_{R^{N}}} \leqslant C h^{l} \sum_{j} \mid \partial_{h}^{l e} j_{\left.v\right|_{\Omega_{1}}} .\right.
\end{aligned}
$$

Together the estimates for I and II prove the lemma.

As an illustration, consider the case when a two-point boundary value problem for a second order ordinary differential equation (or a second order parabolic problem) has been approximately solved using cubic splines on a mesh which is uniform in the interior, so that $O\left(h^{6}\right)$ interior estimates for the difference quotients of the error are available in norms of order -2 . Then the above theory (with $l=2, p=3, r=4$ ) shows that $O\left(h^{6}\right)$ approximations of for instance $u, d u / d x$ and $d^{2} u / d x^{2}$ can be obtained using the trigonometric polynomials $k_{23}, k_{33}$ and $k_{43}$. These approximations are 
(with $T_{\eta} v(x)=v(x-\eta)$ )

$$
\begin{aligned}
& \left(\frac{37}{30}-\frac{23}{180}\left(T_{h}+T_{-h}\right)+\frac{1}{90}\left(T_{2 h}+T_{-2 h}\right)\right) \psi_{(2), h} * u_{h}, \\
& \left(\frac{437}{320}-\frac{97}{480}\left(T_{h}+T_{-h}\right)+\frac{37}{1920}\left(T_{2 h}+T_{-2 h}\right)\right) \psi_{(2), h} * \partial_{h} u_{h} \\
& \left(\frac{181}{120}-\frac{17}{60}\left(T_{h}+T_{-h}\right)+\frac{7}{240}\left(T_{2 h}+T_{-2 h}\right)\right) \psi_{(2), h} * \partial_{h}^{2} u_{h} .
\end{aligned}
$$

Notice that these approximations can easily be expressed in terms of the coefficients of $u_{n}$ with respect to a basis in the finite dimensional approximating space and the convolutions of $\psi_{(2), h}$ with these basis functions. In the particular case of smooth $\left(C^{2}\right)$ cubic splines these convolutions are translates of $\psi_{(6), h}=\psi_{(2), n} * \psi_{(4), n}$.

\section{Department of Mathematics}

Chalmers University of Technology and University of Göteborg

Göteborg, Sweden

1. J. H. BRAMBLE, J. A. NITSCHE \& A. H. SCHATZ, "Maximum-norm interior estimates for Ritz-Galerkin methods," Math. Comp., v. 29, 1975, pp. 677-688.

2. J. H. BRAMBLE \& A. H. SCHATZ, "Higher order local accuracy by averaging in the finite element method," Math. Comp., v. 31, 1977, pp. 94-111.

3. J. H. BRAMBLE, A. H. SCHATZ, V. THOMÉE \& L. B. WAHLBIN, "Some convergence estimates for semidiscrete Galerkin type approximations for parabolic equations," SIAM J. Numer. Anal. (To appear.)

4. PH. BRENNER, V. THOMÉE \& L. B. WAHLBIN, Besov Spaces and Applications to Difference Methods for Initial Value Problems, Lecture Notes in Math., vol. 434, Springer-Verlag, Berlin and New York, 1975. 